\title{
Impact of early life experience on adult health.
}

\author{
David T. Denhardt* \\ Faculty of Emeritus, Division of Life Sciences, Rutgers University, Piscataway, NJ 088854, USA
}

\begin{abstract}
Early life stress (ELS) causes multiple epigenetic modifications (e.g. methylation of genes and histones) in a developing child's genome. Early life stress comes in various forms: Serious physical and mental abuse for example by parents, relatives, or caregivers; a poor social environment involving drug addicts and gang warfare; or loss of all parents and close relatives. This brief review is an attempt to update the information in the above-mentioned opus with a focus on in part on events in the brain and emphasizes that dissociable domains compose executive function.
\end{abstract}

Keywords: Early life stress, Epigenetics, Glucocorticoids.

Accepted on August 11, 2017

\section{Introduction}

Details how ELS may have long-term effects on the individual, in part mediated by methylation of the exon $1 \mathrm{~F}$ variant (site of binding of the transcription factor NGF1-A) of the glucocorticoid receptor $\mathrm{Nr} 3 \mathrm{c} 1$, in the hippocampus, at least in some individuals who later committed suicide are reviewed by Turecki and Meaney $[1,2]$. In a complex way ELS impacts on a set of higher order cognitive processes that enable individuals to orient towards the future, demonstrate self-control, and complete goal-directed behavior encompassed under the term executive function [3]. This includes impulse control, response inhibition, attention, working memory, cognitive flexibility, planning, judgement and decision making. Cowan et al. and colleagues have reviewed possible mechanisms regarding how early-life adversity could impact descendants. Exploratory behavior, curiosity, and an urge for adventure (to explore) may also be related traits, but are poorly understood [4].

\section{Recent Research}

Jawahar et al. has provided a detailed analysis of the current situation, which is complicated by the variety of protocols used (in rodents) to induce ELS [5]. Many genes within the HPA (hypothalamus, pituitary, adrenal) axis are activated and BDNF (bone-derived neurotrophic factor) is produced, which stimulates glucocorticoid production that in turn actives a plethora of downstream genes (the "fight or flight" response) The serotonin transporter, 5-HTT, the estrogen receptor $\alpha$, glutamate decarboxylase 1, and Reelin (involved in neuron migration) are involved according to a variety of reports. A comparison of a group of children raised in an institution with a group raised by their parents revealed differences in whole genome methylation as assessed in peripheral lymphocytes, which are representative of many tissues in the body [6]. In this extensive study $815 \mathrm{CpG}$ sites showed increased methylation and 99 showed decreased methylation. Rodgers et al. reported that sperm of chronically stressed male mice carry certain miRNAs that reduce HPA axis reactivity, "recapitulating" the effect of paternal stress [7].

Others have shown that epigenetic marks found in male mice exposed to malnutrition or drugs of abuse can be passed on to offspring. Murphy et al. quantified differential methylation of regulatory regions of imprinted genes in human cord blood and other tissues; these included SGCE/PEG10 and H19, which are imprinted by only one of the parents [8]. PEG10 (paternally expressed gene 10) functions in trophoblast proliferation and promotes trophoblast invasion n. H19 is a lc nRNA (long noncoding RNA) that contains within it the sequence encoding the micro RNA miR-675, which together H19 are important in controlling many physiological functions, including the epithelial/mesenchymal transitions in both directions.

\section{Discussion}

Poor quality maternal diet (in mice) and subsequent gestational growth disturbances contribute to the etiology of attention deficit hyperactivity disorder (ADHD), autism spectrum disorder (ASD), schizophrenia and abnormalities in executive function. These were linked in part to transcriptional changes in four epigenetic modulators: DNMT1 (DNA methyltransferase), COMT (catechol-O-methyltransferase), ORPD1 ( $\delta$-opioid receptor), and CNR1 (cannabinoid receptor 1) [9].

As detailed recently by Allan et al. considerable evidence suggests that executive function promotes a wide range of healthy behaviors, including increased physical, intellectual and social activity and a sensible low-fat diet with minimal smoking or drinking [10]. 5 hydroxy tryptophan-2a (5HT2a) is a serotonin receptor involved in executive function and is located in the prefrontal cortex just behind the forehead. It is essential in planning complex behavior, personality expression, decision making and moderating social behavior. The postsynaptic serotonin 2A receptor, 5-HT2AR, is the most abundant subtype modulating cortical network activity; it induces synchronized spiking of single units and neuronal ensembles, known as wave oscillation synchronization. It also has a modulatory effect on dopaminergic signaling, another important player in executive function. Importantly, dopamine is now recognized as a potential treatment target for many neuropsychiatric disorders [11].

\section{Conclusion}

Metabolic pathways linked to mental health disorders (e.g. social anxiety disorder, a common and disabling disorder, appears to 
result from decreased methylation of the oxytocin receptor, OXTR. Oxytocin, a 9-amino acid neuropeptide, is known to increase positive social interactions and pair bonding. Evidence strongly suggests that one cause of SAD is the result of OXTR hypomethylation, conferring increased OXTR expression [12].

\section{Grant Support}

This research was self-funded by DTD, who takes full responsibility for its content.

\section{References}

1. Denhardt DT. Effect of stress on human biology: Epigenetics, adaptation, inheritance, and social significance. J Cell Physiol. 2017.

2. Turecki G, Meaney MJ. Effects of the social environment and stress on glucocorticoid receptor gene methylation: A systematic review. 2016;79:87-96.

3. Logue SF, Gould TJ. The neural and genetic basis of executive function: attention, cognitive flexibility, and response inhibition. Pharmacol Biochem Behav. 2014;123:45-54.

4. Cowan CS, Callaghan BL, Kan JM, et al. The lasting impact of early-life adversity on individuals and their descendants: Potential mechanisms and hope for intervention. Genes Brain Behav. 2016;15:155-68.

5. Jawahar, MC, Murgatroyd, C, Harrison, EL, et al. Epigenetic alterations following early postnatal stress: a review on novel aetiological mechanisms of common psychiatric disorders. Clin Epigenetics. 2015;14:122.
6. Naumova OY, Lee M, Koposov R, et al. Differential patterns of whole-genome DNA methylation in institutionalized children and children raised by their biological parents. Dev Psychopathol. 2012;24:143-55.

7. Rodgers AB, Morgan CP, Leu NA, et al. Transgenerational epigenetic programming via sperm microRNA recapitulates effects of paternal stress. Proc Natl Acad Sci USA. 2015;112:13699-704.

8. Murphy SK, Huang Z, Hoyo C. Differentially methylated regions of imprinted genes in prenatal, perinatal and postnatal human tissues. (2012) PLoS One. 7

9. Grissom NM, Herdt CT, Desilets J, et al. Dissociable deficits of executive function caused by gestational adversity are linked to specific transcriptional changes in the prefrontal cortex. Neuropsychopharmacology. 2015; 40:1353-63.

10. Allan JL, McMinn D, Daly M. A Bidirectional Relationship between executive function and health behavior: Evidence, implications, and future directions. Front Neurosci. 2016;10: 386 .

11. Aznar S, Hervig MES. The 5-HT2A serotonin receptor in executive function: Implications for neuropsychiatric and neurodegenerative diseases. Neurosci Biobehav Rev. 2016;64:63-82.

12. Ziegler C, Dannlowski U, Bräuer D, et al. Oxytocin receptor gene methylation: converging multilevel evidence for a role in social anxiety. Neuropsycho-Pharmacology. 2015;40:1528-38.

\section{*Correspondence to:}

David T. Denhardt

Professor of Emeritus

Division of Life Sciences

111 Checkerberry Lane, Ensboro, NC 27455

Gre Rutgers University, Piscataway

NJ 088854, USA

Tel: (732) 445-4569

E-mail: Denhardt@dls.rutgers.edu 